\title{
Application of $\ell_{p}$ norm regularisation techniques in the synthesis of indoor tyre pass-by noise with the inverse method
}

\author{
Athanasios Papaioannou*, Stephen J. Elliott, Jordan Cheer \\ Institute of Sound and Vibration Research, University of Southampton, Highfield, Southampton SO17 1BJ, United Kingdom
}

\begin{abstract}
Pass-by noise measurements can nowadays be realised in a laboratory environment with a microphone array and a stationary vehicle on a rolling road, according to ISO - 362-1:2016. Within this indoor testing procedure, the contributions from the various noise sources in a car can be estimated, with a set of microphones close to the various sources, and the pass-by noise then synthesised. The methods described in this paper use measured near field pressure data close to a rolling tyre at $50 \mathrm{~km} / \mathrm{h}$ to estimate virtual source strength, using the inverse method, and the synthesised far field tyre noise is then compared with the one measured directly at a far field microphone array. A combination of $\ell_{1}$ norm and $\ell_{2}$ norm regularisation methods are investigated to optimise the equivalent source position and strength and, subsequently, the far field pass-by noise pressure estimates through the frequency range of interest. It is shown that by optimising the positions of 4 equivalent sources, acceptable representations of the measured far field spectra can be synthesized.
\end{abstract}

Keywords: pass-by noise, tyre noise, inverse method, $\ell_{1}$ norm, regularisation

\section{Introduction}

The exterior sound emission from a vehicle is an increasingly important criterion for the homologation of road vehicles. Until recently, pass-by noise has been measured outdoors, but can now be measured in an indoor environment with a far field microphone array and a stationary vehicle on a rolling road, according

5 to ISO-362:2016 [1]. This standard also describes pass-by noise testing systems, using a roller bench in a semi-anechoic room, to increase the total robustness and repeatability of the measurements and there also exists the ability to quantify the various car noise source contributions. This is described as the pass-by noise contribution analysis and is nowadays widely used in the field of vehicle noise control as it gives further insight into the total noise disturbance [2].

Tyre noise has become one of the major sources of pass-by noise due to considerable efforts in reducing engine noise and increasing use of electric vehicles. The indoor tyre pass-by noise contribution can be estimated in fully operational conditions by using a set of additional microphones close to the real car sources using the concept of acoustic transfer path analysis (TPA) [3-4]. The tyre is approximated by a set of equivalent sources and the acoustic transfer responses are measured between these sources and all

15 near field microphone positions, to give a full matrix of coupling coefficients. The various equivalent source strengths are then estimated by combining the pressure responses measured by the near field microphone array and the inverse of the transfer response matrix. A new set of acoustic transfer functions is then measured between the source positions and a linear microphone array $7.5 \mathrm{~m}$ away from the car, which is then used to synthesise the far field acoustic pressure.

A number of studies have used the noise contribution analysis to quantify the indoor tyre pass-by noise, along with all the various noise source contributions in fully operational conditions [5-10]. A slightly different

\footnotetext{
*Corresponding author. Tel.: +44 (0)23 80594932 .

Email address: A.Papaioannou@soton.ac.uk (Athanasios Papaioannou)
} 
variation is proposed by Janssens et al, where the phase information is omitted from the measured spectra by assuming an energetic, power-based approach to the traditional formulation [2]. Research has also been done on the prediction of tyre pass-by noise by combining the indoor pass-by noise contribution analysis outdoor measurements [11]. In this previous work, the indoor tyre noise contribution is estimated by assuming a small number of uncorrelated monopole sources very close to the leading and trailing edge of each tyre, using double this number of near field microphones in close proximity to the tyre. The positions of the equivalent point sources are, therefore, chosen based on experience and the knowledge that the most dominant tyre noise sources are situated at the tyre-road contact area [12], while the number of sources is kept low to minimise the duration and complexity of the measurement sessions.

Different applications of the inverse method to the synthesis of far field tyre noise have also investigated alternative distributions of equivalent sources. In [13] and [14], the tyre is split into a multitude of segments represented by a number of predefined source positions to synthesise the far field tyre noise, which requires a considerable number of microphones, measurements and post-processing time. In [15], a collection of

35 different equivalent source distributions is investigated starting from only a few up to 40 uncorrelated monopoles to synthesise tyre noise in the far field from near field measurements. However, while conclusions can be drawn as to the number of equivalent uncorrelated sources needed for an accurate far field pressure reconstruction, the source positions are not optimised in order to achieve the best possible accuracy under the given operational conditions, but rather chosen beforehand and tested for their ability to accurately reconstruct the sound field.

With the aim of overcoming this limitation, the work presented here utilises two $\ell_{1}$ norm regularisation methods to select a few equivalent source locations out of a larger number of possible locations using compressive sensing techniques, applied in real-life data measured in the semi-anechoic chamber of Applus+ IDIADA. The novelty of this work is that the methods utilise the sparsity-promoting properties of the $\ell_{1}$ norm

45 to choose optimised equivalent source distribution for a given number of sources using the inverse method and enhance the accuracy of the tyre noise contribution far field synthesis in an indoor pass-by noise context. Different numbers of equivalent source positions are assumed, while a $\ell_{2}$ norm regularisation technique is also deployed to improve the reconstruction accuracy at lower frequencies when using a larger number of sources. The aim of this paper is therefore to investigate the application of regularisation techniques to optimise the equivalent source geometry and far field synthesised spectra for a given number of sources without assuming prior knowledge of the noise generating mechanisms of the tyre. Although prior knowledge of the transfer functions between the source positions and the microphone array positions is still required, a fast and robust selection of source positions can be performed compared to an exhaustive search of the various combinations of equivalent source positions which grows geometrically in time with increasing number of assumed source positions. It is noted, however, that the results obtained in this paper are strictly valid only for the given measurement conditions, and further work should be done to assess the validity of the results in other operational conditions.

In Section 2, the theory of the inverse method used for the tyre noise synthesis including the different regularisation strategies is covered, while, in Section 3, the measurement set-up is presented. The corresponding results are discussed in Section 4, while the conclusions drawn are covered in Section 5.

\section{Theory and formulation}

The synthesis of the far field pressure with use of near field measured spectra is a two-step process. It consists of an inverse method where the equivalent source strengths on a tyre are reconstructed by using the near field spectra and a forward problem where the source strengths are used to synthesise the pressure in the far field. The accuracy of the synthesis is largely dependent on the accuracy of the inverse method in the first step, which is correspondingly determined by the conditioning of the transfer matrix to be inverted [16-17]. This matrix is often ill-conditioned and, subsequently, susceptible to errors associated with noise and uncertainties inherited through the measurements.

The susceptibility of the problem to errors, however, can be controlled by selecting the geometry of the problem, that is the spatial sampling of the source and the microphone array as a function of frequency 
$[18,19]$. In this paper, two $\ell_{1}$ norm regularisation techniques are investigated which utilise the sparsitypromoting properties of the $\ell_{1}$ norm to directly associate the choice of the equivalent source positions with the cost function to be minimised. The first technique is a Compressive Sensing technique [20,21] which has previously been used in near-field acoustic holography (NAH) [22-24] and beamforming applications [25], while the second one is its generalisation for the simultaneous recovery of signals with shared sparsity properties and has been used in source localisation [26] and other applications [27-30]. The approach is to identify the positioning of a small number of equivalent sources that can then be used independently to repeat measurements, thus minimising the instrumentation and complexity.

Once the equivalent source positions are chosen for various numbers of source elements, the inverse problem is then solved using the pseudo-inverse that is formed using the equivalent source positions chosen by the compressive sensing techniques. A standard $\ell_{2}$ norm (Tikhonov) regularisation technique is finally further used to alleviate the effect of poor conditioning at lower frequencies and the final source strengths are then used to synthesise the far field pressure and compare it to the one measured directly.

\subsection{Use of $\ell_{1}$ norm regularisation at each frequency}

As a first step, the pressure is assumed to be measured with a near field array of $M$ microphones. Tyre noise is mostly broadband and has a time dependence that can be regarded as random with stationary statistical properties at a given road speed. Therefore, the spectra are estimated by defining the Fourier transform from a series of finite length histories of measured data [16]. For example, the discrete Fourier spectrum at the $m$ th microphone is defined by

$$
p_{\text {near }, m}(k)=\sum_{n=0}^{N-1} p_{m}(n) \mathrm{e}^{-\mathrm{i} \omega_{k} n T}
$$

where $p_{m}$ is the sequence of $\mathrm{N}$ pressure samples, $T$ is the period and $\omega_{k}=\frac{2 \pi k}{N T}$. These spectra will in practice be contaminated by errors and noise. The modulus squared Fourier transform can be smoothed to give the power spectral density either by averaging the modulus squared spectra over multiple blocks of data in the time domain, or by averaging the modulus squares spectra across multiple frequency bins in the Fourier transform for a single large set of time-domain data [31]. The tyre radiation is approximated by

${ }_{95} N$ monopoles distributed over its radiating surface with complex source strengths $\mathbf{q}$, while their pressure contributions to the near-field are estimated by means of a frequency response function matrix $\mathbf{G}_{\text {near }}$ of size $M \mathrm{x} N$ which in practice should also be measured. This is because waves reflected and scattered within the measurement environment are only properly captured by measuring the $\mathbf{G}_{\text {near }}$ with the consequence that using the same $\mathbf{G}_{\text {near }}$ may not produce the same results for another vehicle. Therefore, the equation describing the system at a given frequency is

$$
\mathbf{p}_{\text {near }}=\mathbf{G}_{\text {near }} \mathbf{q}+\mathbf{e}
$$

where $\mathbf{p}_{\text {near }}$ is a vector of $p_{\text {near, } m}$ and $\mathbf{e}$ is a vector of complex errors.

Unlike conventional methods, in this configuration the total number of possible equivalent source positions can be greater than the number of near field microphones (i.e. $N>M$ ). In the conventional least-squares case, a full-rank solution would imply that the system has either no solution or an infinite number of solutions. However, sparsity, herein introduced by adding a penalty in the $\ell_{1}$ norm of the source strength vector $\mathbf{q}$, forces it to be decomposed as a combination of a number of elements equal to $N_{f}$, which is a subset of the number of source positions $N$ (i.e. $N \geq N_{f}$ ) [23]. This decomposition can either be exact, meaning that the source strengths of the rest of the source positions are set to zero, or approximate, meaning that they are highly compressed to a level where they can be regarded as negligible. By enforcing an acceptable level of sparsity with a constraint on the $\ell_{1}$ norm of $\mathbf{q}$, a number of non-zero point sources smaller or equal to the number of near field microphones is finally used $\left(N>M \geq N_{f}\right)[26]$ and the final system of equations becomes resolvable in the constrained minimisation problem

$$
\min _{\mathbf{q}}\left\|\mathbf{G}_{\text {near }} \mathbf{q}-\mathbf{p}_{\text {near }}\right\|_{2}^{2} \text { subject to }\|\mathbf{q}\|_{1} \leq \delta
$$


where $\delta$ is a positive scalar, \|\|$_{2}^{2}$ denotes the squared $\ell_{2}$ norm and \|\|$_{1}$ denotes the $\ell_{1}$ norm, the solution of which is a convex problem [23].

The level of sparsity in the source strength vector $\mathbf{q}$ is adjusted by tuning the regularisation parameter $\delta$. This parameter directly represents the $\ell_{1}$ norm, that is the sum of the absolute values of the source strength vector at that frequency. Therefore, the lower the $\delta$, the more it is going to enforce sparsity in the solution and thus a smaller number of non-zero equivalent sources will be used in the inverse problem. The careful selection of $\delta$ gives the opportunity to choose the number of sources with which the source reconstruction is realised at each frequency and their distribution from the grid of candidate sources is optimised to minimise the least-squares linear problem in Eq. (3).

The $\ell_{1}$ norm is used as a convex alternative to the non-convex sparse minimisation of the $\ell_{0}$ norm, which is essentially a direct selection of the number of non-zero source strength positions [23]. Since the $\ell_{1}$ norm regularisation cannot be expressed in closed form, it has no analytical solution and, therefore, iterative numerical methods are used to solve it. Numerous algorithms exist (interior point algorithms, iterative reweighted least squares, gradient projection etc.) and several toolboxes are available, such as CVX [32] and SPGL1 [33,34]. The latter is the one used in this paper.

This regularisation technique can normally provide an estimate of the source strength distribution without computing the ill-conditioned pseudo-inverse of the transfer matrix $\mathbf{G}_{\text {near }}$. In this paper, the technique is used to select a small number of equivalent source positions to minimise the measurement complexity and instrumentation. Therefore, once the equivalent source positioning is identified at each frequency, the inverse problem is then solved using the pseudo-inverse formed between the non-zero source components and the near-field microphones.

\subsection{Use of $\ell_{1}$ norm regularisation over a range of frequencies}

The $\ell_{1}$ norm regularisation, as presented in Sec. (2.1), can be used to give the same number of equivalent sources at each individual frequency, however it does not guarantee that those sources will be located at the same positions over a range of frequencies. This is impractical for synthesis since it implies the need for measurements at all the various positions used over the frequency range of interest and increases the amount of instrumentation, complexity and post-processing time. Therefore, what is additionally needed is a method that can predict a fixed equivalent source positioning, that is the same few equivalent source positions over the frequency range of interest. If the responses are measured in discrete frequency bins, $\omega_{k}$, we assume that the frequency range of interest is from $k=K_{1}$ to $K_{2}$, which may be the whole frequency range of interest or just $1 / 3$ octave bands. The constrained minimisation problem of Eq. (3) is thus modified as below

$$
\min _{\mathbf{Q}} \sum_{k=K_{1}}^{K_{2}}\left\|\mathbf{G}_{\text {near }}\left(\omega_{k}\right) \mathbf{q}\left(\omega_{k}\right)-\mathbf{p}_{\text {near }}\left(\omega_{k}\right)\right\|_{2}^{2} \text { subject to }\|\mathbf{Q}\|_{1,2} \leq \epsilon
$$

where the subscript $k$ corresponds to the discrete frequency lines and $\mathbf{Q}$ is a $N \times K$ matrix whose columns are the source strength vectors $\mathbf{q}\left(\omega_{k}\right)$ at each frequency line $k$. The $n$th row of $\mathbf{Q}$ is then $\mathbf{q}^{n}=\left[q^{n}\left[K_{1}\right], \ldots, q^{n}\left[K_{2}\right]\right]$ and the mixed $\ell_{1,2}$ norm of $\mathbf{Q}$ is then defined as

$$
\|\mathbf{Q}\|_{1,2}=\sum_{n=1}^{N}\left(\left\|\mathbf{q}^{n}\right\|_{2}\right)^{1}=\sum_{n=1}^{N}\left(\sum_{k=K_{1}}^{K_{2}}\left|q^{n}[k]\right|^{2}\right)^{1 / 2}
$$

This mixed $\ell_{1,2}$ norm is suitable in this application as it promotes a common sparsity profile for the various frequency lines, that is the non-zero source strength positions are fixed over frequency [34]. The level of sparsity which translates to the number of non-zero equivalent source positions is controlled by the regularisation parameter $\epsilon$, similarly to the way this is done with $\delta$ at each distinct frequency line in Sec. 2.1. Note that this technique effectively uses the second method of estimating power spectral density described above, where the mean square value of the Fourier transform is averaged over the bins within the bandwidth considered. This constrained optimisation can, therefore, yield source strength vectors $\mathbf{q}$ which share the same sparsity pattern with respect to the source positions and minimise the sum of the least-squares linear 
problem over frequency. This method belongs to the family of the joint sparse recovery problems and can be solved using different variations of the mixed $\ell_{p, q}$ norm. The SPGL1 toolbox is again used, as in Sec. (2.1), to solve the problem, while the use of the mixed norm is more fully explained in [35]. The generalisation of the single frequency least squares error to the sum of the errors over the frequency range is done by replacing $\mathbf{G}_{\text {near }}$ with a diagonal block of matrices which contains the various $\mathbf{G}_{\text {near }}\left(\omega_{k}\right)$ at each frequency line $k$. The formulation solved and used in Eq. (4) is the one of the Lasso technique, which minimises the residual norm subject to the $\ell_{1}$ norm of the coefficients being no greater than a given regularisation parameter. This is an alternative to the Basis-Pursuit-Denoising formulation, which minimises the $\ell_{1}$ norm of the coefficients subject to the residual norm no greater than a given regularisation parameter [29]. Similar to Sec. (2.1), once the equivalent source positioning is identified over the frequency range of interest, the inverse problem is then solved using the pseudo-inverse $\mathbf{G}_{\text {near }}^{\prime}{ }^{+}$formed between the non-zero source components and the near-field microphones.

\subsection{Additional use of $\ell_{2}$ norm regularisation}

After the use of $\ell_{1}$ norm regularisation technique, the problem is reduced to a determined or overdetermined one with $\mathbf{q}^{\prime}$ the size of $N_{f} \mathrm{x} 1$ and the reduced transfer response function matrix $\mathbf{G}_{\text {near }}^{\prime}$ the size of $M \mathrm{x} N_{f}$, where $M \geq N_{f}$. However, although the synthesis accuracy is controlled to an extent by the choice of the source geometry for a specific number of sources, a low frequency correction is often still needed, especially when an increasing number of equivalent sources is used and the condition number rises at lower frequencies [18]. Therefore, once the equivalent source distribution is calculated using the $\ell_{1}$ norm penalty terms, a conventional $\ell_{2}$ norm regularisation is performed in the inverse problem by additionally minimising the $\ell_{2}$ norm of the source strengths $\mathbf{q}^{\prime}$

$$
\min _{\mathbf{q}^{\prime}}\left\|\mathbf{G}_{\text {near }}^{\prime} \mathbf{q}^{\prime}-\mathbf{p}_{\text {near }}\right\|_{2}^{2}+\beta\left\|\mathbf{q}^{\prime}\right\|_{2}^{2}
$$

where $\beta$ is a positive scalar. The source strengths are then given by

$$
\mathbf{q}^{\prime}=\mathbf{G}_{\text {near }, \beta}^{\prime+} \mathbf{p}_{\text {near }}
$$

and the regularised pseudo-inverse matrix then takes the form

$$
\mathbf{G}_{\text {near }, \beta}^{\prime}{ }^{+}=\left[\mathbf{G}_{\text {near }}^{\prime}{ }^{\mathrm{H}} \mathbf{G}_{\text {near }}^{\prime}+\beta \mathbf{I}\right]^{-1} \mathbf{G}_{\text {near }}^{\prime}{ }^{\mathrm{H}}
$$

where ${ }^{\mathrm{H}}$ is the Hermitian transpose operator. The parameter $\beta$ discriminates against small singular values, reducing the magnitude of their inverse and softening them [18]. This regularisation method is chosen herein instead of singular value discarding, which performs a harsh cut-off of the smallest singular values of $\mathbf{G}_{\text {near }}^{\prime}$ and can reduce the spatial resolution at higher frequencies [17]. It should be noted that the power spectral matrix for the equivalent source strengths

$$
\mathbf{S}_{\mathbf{q q}}=\mathbf{G}_{\text {near }, \beta}^{\prime}{ }^{+} \mathbf{S}_{\mathbf{p p}_{\text {near }}} \mathbf{G}_{\text {near }, \beta}^{\prime+\mathrm{H}}
$$

where $\mathbf{S}_{\mathbf{p} \mathbf{p}_{\text {near }}}$, the power spectral matrix of the near field pressure, is not necessarily diagonal, demonstrating that the equivalent sources do not need to be uncorrelated.

\subsection{Far field tyre pressure synthesis}

An estimate of the pressures in the linear far field array is then given by

$$
\tilde{\mathbf{p}}_{\mathrm{far}}=\mathrm{G}_{\mathrm{far}}^{\prime} \mathbf{q}^{\prime}
$$

where $\mathbf{G}_{\text {far }}^{\prime}$ is the transfer response matrix connecting the far field microphones and the non-zero equivalent source positions. In practice, this matrix should also be measured as it may differ in other operational conditions. This estimate is finally compared to the exact directly radiated field $\mathbf{p}_{\mathbf{f a r}}$. A diagram illustrating the signals and optimisation processes involved in this Section is given in Fig. 1. 


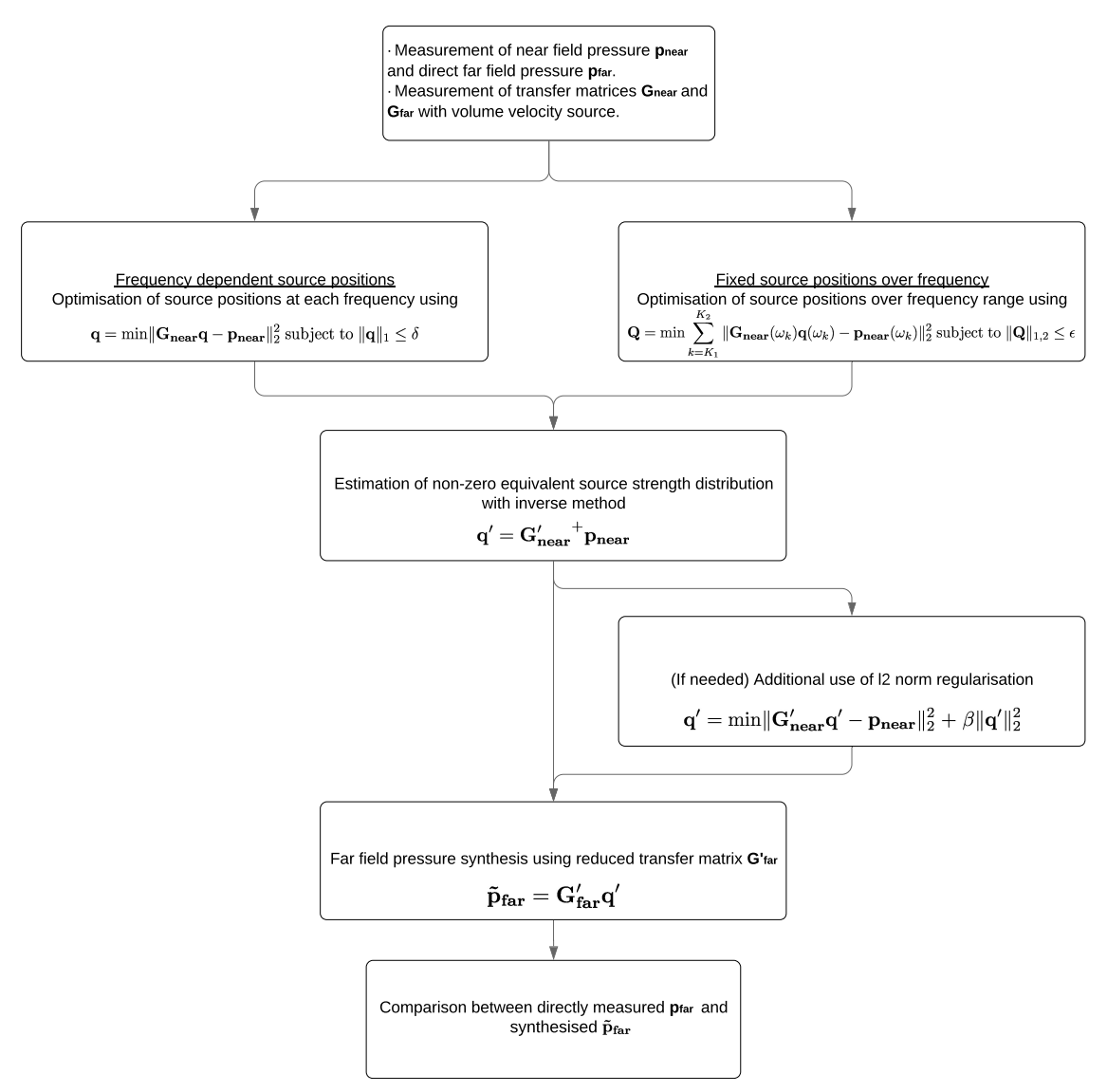

Figure 1: Diagram illustrating the signal acquisition and optimisation process described in Section 2.

\section{Experiments on a real car tyre rolling at $50 \mathrm{~km} / \mathrm{h}$}

The methods described above were tested on a real car tyre source on a rolling road at $50 \mathrm{~km} / \mathrm{h}$. The objectives of the experiment were to test the ability of the $\ell_{1}$ norm regularisation techniques to deliver source geometries with respect to the near field inverse problem and investigate the number of sources and regularisation needed for an accurate far field spectrum synthesis.

The experiment was split into two measurement sessions which took place in the semi-anechoic chamber in Applus+ IDIADA [36]. In both campaigns, a SEAT Ibiza was fixed in the designated area so that the back tyres of type Michelin 175/70R14 (total radius equal to $30 \mathrm{~cm}$ ) were placed on top of the rolling road, controlled by two independent motors, one for each tyre. Fig. 2 shows the fixed car above the rolling road in the IDIADA semi-anechoic chamber.

For the purposes of the measurements described below, only the motor controlling the right rear tyre was driven. The pressure signals were all measured simultaneously, using an NI-DAQmx acquisition system. Each measurement had a duration of $60 \mathrm{~s}$, while the sampling frequency was set to $25,600 \mathrm{~Hz}$.

\subsection{Far field array measurements}

In the first measurement session, $14 \mathrm{PCB}$ microphones were placed in a linear array $4.3 \mathrm{~m}$ away from the car. This distance is smaller than the $7.5 \mathrm{~m}$ suggested within the ISO guidelines [1], but was the maximum possible due to the limitations of the room dimensions whilst keeping a distance of $1.2 \mathrm{~m}$ between the array 


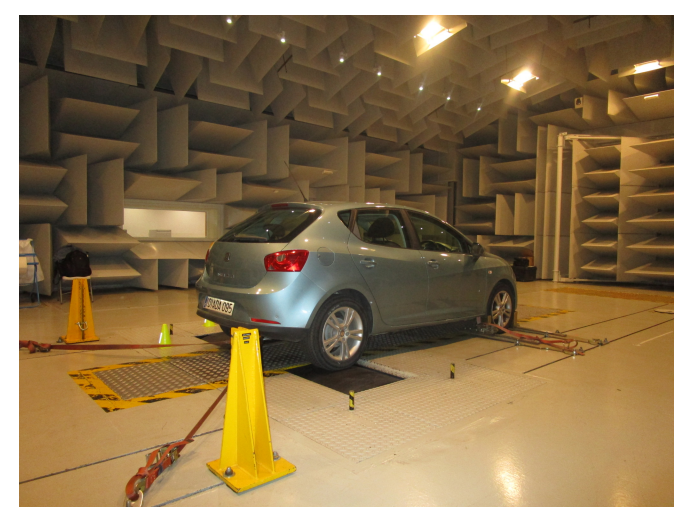

Figure 2: Fixed car in the IDIADA semi-anechoic chamber.

and the wedges to assume free field conditions. The microphone height was set to $0.85 \mathrm{~m}$, adjusted from the $1.2 \mathrm{~m}$ suggested in [1] to be in line with the smaller distance from the car. The microphone spacing was set to $0.9 \mathrm{~m}$, implying a $11.7 \mathrm{~m}$ total array length. In Fig. 3, a schematic of the far field array measurement topology is given, along with a photo from the real measurement topology in the IDIADA semi-anechoic chamber.

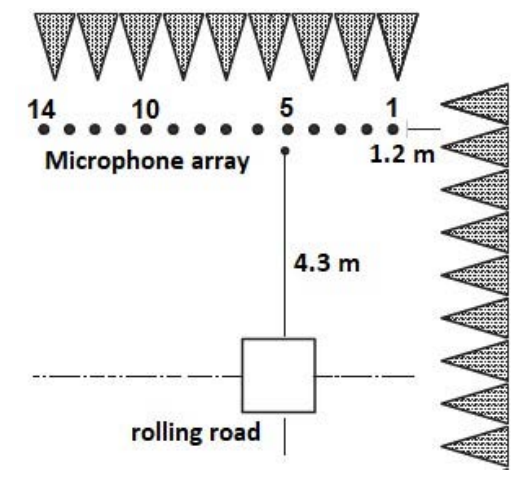

(a)

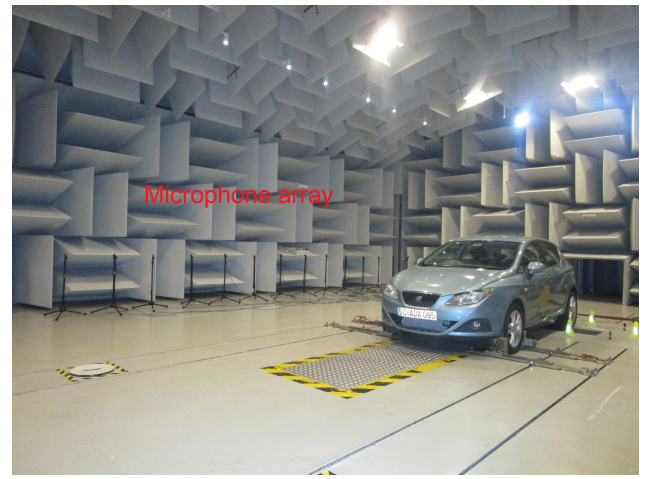

(b)

Figure 3: (a)Schematic of the far field array measurement campaign, (b) Far field measurement campaign in the IDIADA semi-anechoic chamber.

Before moving the car into the chamber, the background noise spectra $\mathbf{p}_{\mathbf{b}, \text { far }}$ was measured in the far field array with the right motor running at $50 \mathrm{~km} / \mathrm{h}$ between 100 and $10000 \mathrm{~Hz}$ in order to quantify the effect of the motor noise. This was found to be approximately $26 \mathrm{~dB}$ less than the noise generated by the tyre and so could be ignored. The car was then placed in the chamber and the right rear tyre pressure spectrum $\mathbf{p}_{\text {far }}$ was measured at the 14 far field microphones for a speed of $50 \mathrm{~km} / \mathrm{h}$.

An omni-directional volume velocity source, covering the range between $100-10000 \mathrm{~Hz}$, supplied by ISVR Consulting [37], was then used to measure the transfer responses between 20 equivalent source positions and the far field array. A hose was attached to the source and it was moved to the various candidate source positions, fixed by a clamp. Fig. 4(a) shows a snapshot of the far field transfer response measurement with the volume velocity source. The source positions were chosen to be radially distributed along the tyre circumference starting from the leading edge towards the trailing edge. The sources located close to the edges were positioned at the centre of the tyre tread, while the rest were located on the side of the tyre due to accessibility. The 20 - equivalent source grid is also given in Fig. 4(b).

A fully coupled matrix of $\mathbf{G}_{\mathbf{f a r}}$, representing the transfer paths between the 14 microphones and the 20 candidate source positions, was calculated at each frequency line over the range of 100 to $10000 \mathrm{~Hz}$. The 


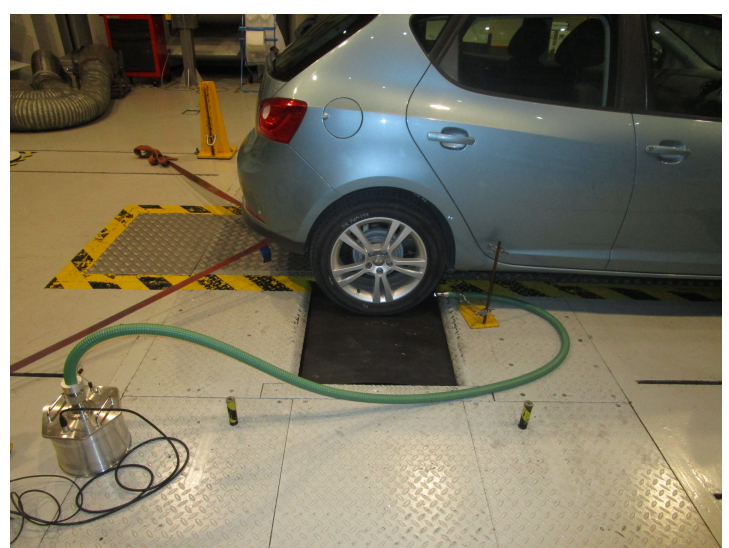

(a)

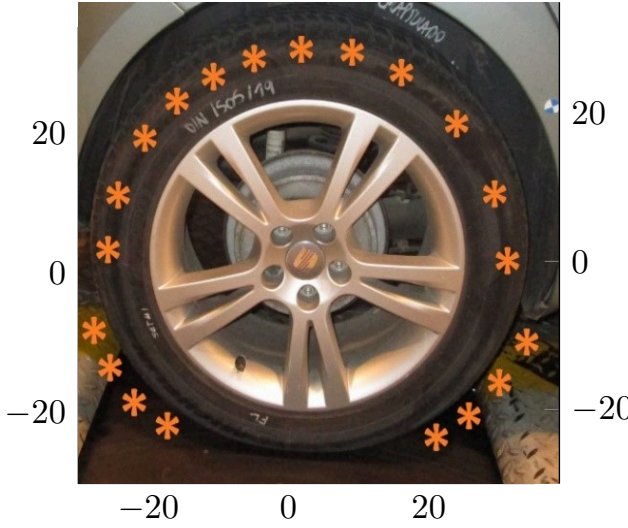

(b)

Figure 4: (a) Measurement of the far field transfer responses between far field microphone array and equivalent source positions with the volume velocity sources, (b) Equivalent source position grid in $\mathrm{cm}$.

Fourier transform of 60 seconds of data was used to calculate the $p_{\text {near }, m}(\omega)$ in Eq. (1) which had 1,536,000 points.

\subsection{Near field array measurements}

In the second measurement session, an array of 15 PCB microphones was placed close to the circumference of the tyre. 14 of those microphones formed a circular array attached on the car chassis $10-15 \mathrm{~cm}$ away from the tyre surface and the last microphone was placed on the tyre side at a distance of $55 \mathrm{~cm}$. The number of microphones was chosen as 15 to be approximately double the maximum number of equivalent sources considered within the regularisation techniques (8 equivalent sources) and maintain the best conditioning possible in the transfer matrix inversion process. Fig. 5 shows the near field array configuration.

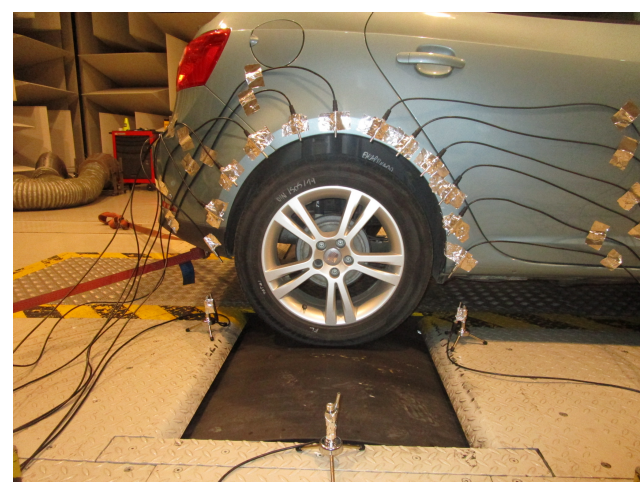

Figure 5: Near field microphone array configuration.

The near field right rear tyre pressure spectrum $\mathbf{p}_{\text {near }}$ was then measured at the 15 near field microphones for an equivalent road speed of $50 \mathrm{~km} / \mathrm{h}$, with the engine off, over the frequency range of 100 to $10000 \mathrm{~Hz}$.

The volume velocity source was then used to measure the transfer responses between the 20 equally spaced equivalent source positions along the tyre circumference and the near field array. A snapshot of the near field transfer response measurements is given in Fig. 6. A fully coupled matrix of $\mathbf{G}_{\text {near }}$, representing the transfer paths between the 15 microphones and the 20 candidate source positions, was therefore calculated at each frequency line over the frequency range of interest $(100$ to $10000 \mathrm{~Hz})$. 


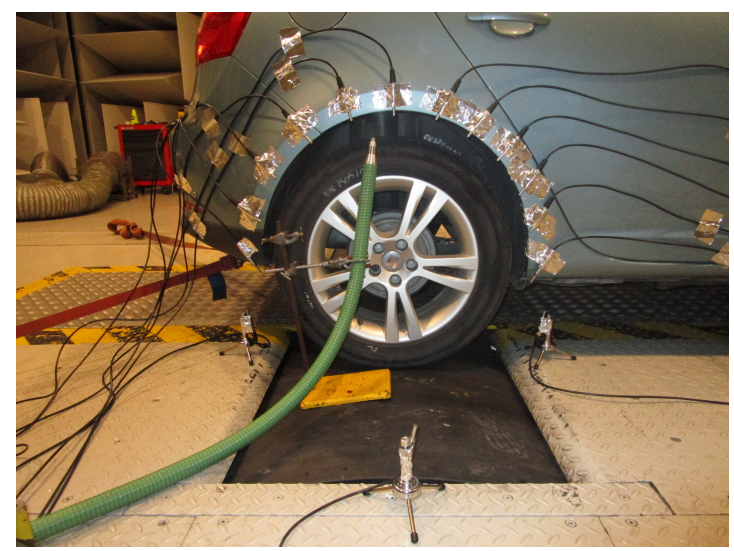

Figure 6: Measurement of the near field transfer responses between near field array and source positions with the volume velocity source.

\section{Results}

A detailed analysis is given below for the synthesis of the $50 \mathrm{~km} / \mathrm{h}$ far field noise spectra highlighting the effect of the regularisation techniques in the synthesised results.

\subsection{Effect of $\ell_{1}$ norm regularisation techniques}

The first step of the analysis is the use of the $\ell_{1}$ norm regularisation techniques to choose the source distribution for either 1,2, 4 or 8 equivalent sources, from the grid of the 20 source positions, and utilise them to synthesise the far field tyre noise pressure. To accomplish that, a selection of the regularisation parameters, as defined in Eq. $(3,4)$, must be done, which, for the case of the $\ell_{1}$ norm regularisation at each frequency, is a frequency dependent scalar, while, for the case of the $\ell_{1}$ norm regularisation over the frequency range, is a single scalar. In Fig. 7, the relationship between regularisation parameters $\delta, \epsilon$ and the number of non - negligible sources is given, where the source strengths were considered negligible if their amplitude was $20 \mathrm{~dB}$ less than the maximum one.

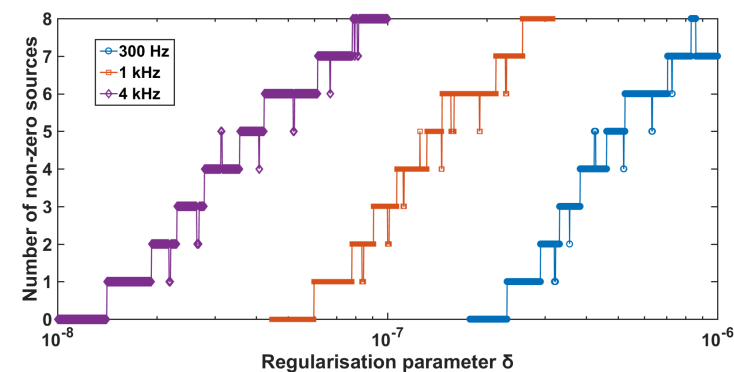

(a)

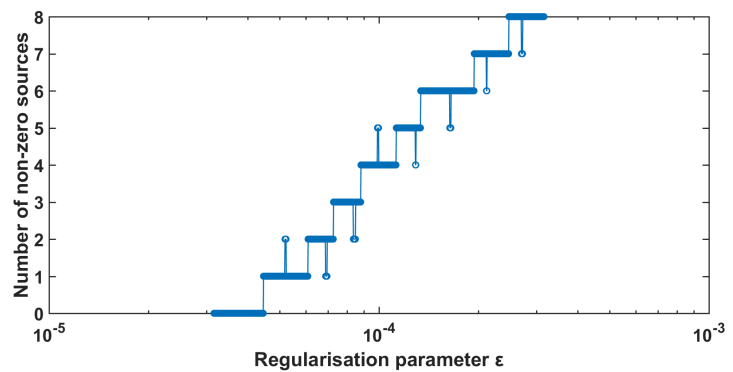

(b)

Figure 7: Relationship between regularisation parameters (a) at individual frequencies $\delta$, (b) average over all frequencies $\epsilon$ and number of non-negligible sources.

Fig. 7(a) shows the relationship between the regularisation parameter $\delta$ and the number of non-negligible sources used in the inverse problem of Eq. (3) at frequencies of $300 \mathrm{~Hz}, 1 \mathrm{kHz}$ and $4 \mathrm{kHz}$. It is seen that the use of a smaller regularisation parameter results in the selection of a smaller number of sources as expected. A few outliers are seen to occur between adjacent numbers of non-negligible sources which is due to the fact that the algorithm used converges to solutions with different number of sources for specific values of the regularisation parameters. It is also shown that an increase in frequency results in a decrease in the value of 
$\delta$ required to enforce the same level of sparsity, which suggests a decrease in the source strength amplitudes with increasing frequency. This is probably related to the fact that the short wavelength components do not radiate efficiently. Fig. 7(b) shows the relationship between the regularisation parameter $\epsilon$ and the number of non-negligible sources used in the frequency - averaged inverse problem of Eq. (4). In this case, $\epsilon$ represents the sum of the absolute values of the source strength vectors over the frequency range of interest, therefore it is not frequency dependent. It is again shown that by decreasing $\epsilon$, a smaller number of non-zero sources is selected. For both regularisation strategies, the regularisation parameter that minimises the errors defined in Eq. (3) and (4) is chosen within the range of the parameter which results in the use of a chosen number of non-zero sources. This is done for $1,2,4$ and 8 equivalent sources. Fig. 8 shows the equivalent source positions using the frequency dependent $\ell_{1}$ norm regularisation at $300 \mathrm{~Hz}, 1 \mathrm{kHz}$ and $4 \mathrm{kHz}$ and the frequency - averaged $\ell_{1}$ norm regularisation for the cases of $1,2,4$ and 8 sources.

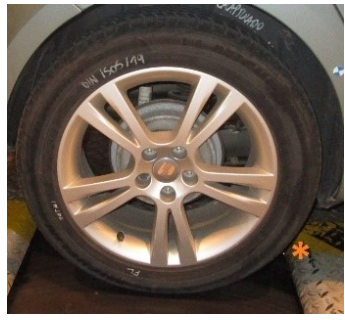

(a)

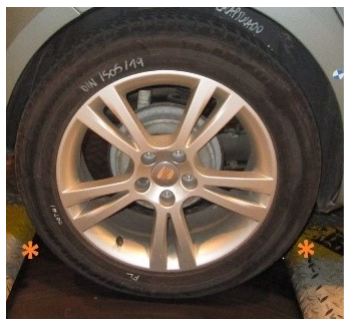

(b)

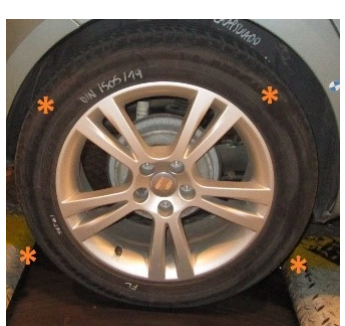

(c)

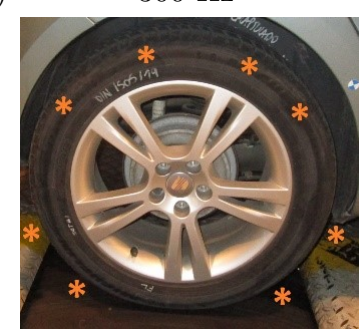

(d)

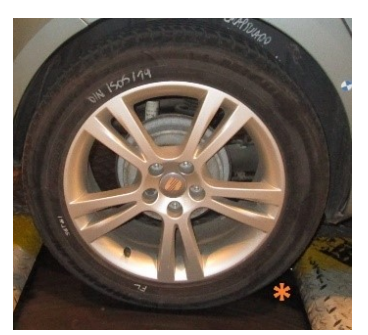

$1 \mathrm{kHz}$

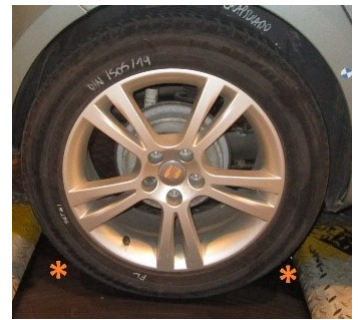

$1 \mathrm{kHz}$

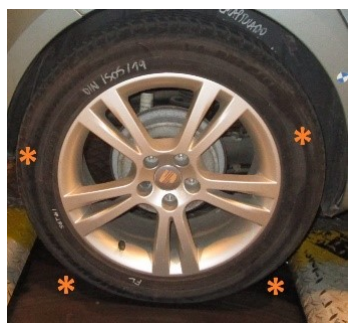

$1 \mathrm{kHz}$

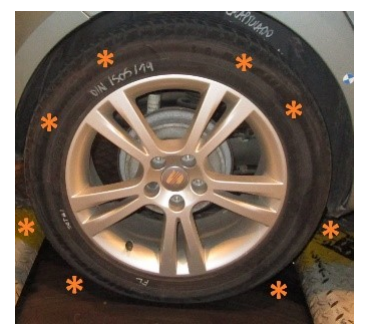

$1 \mathrm{kHz}$

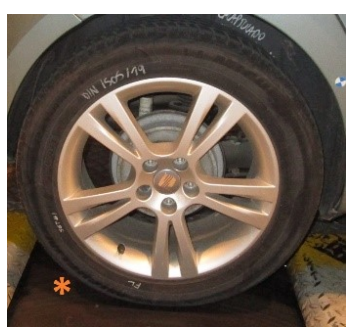

$4 \mathrm{kHz}$

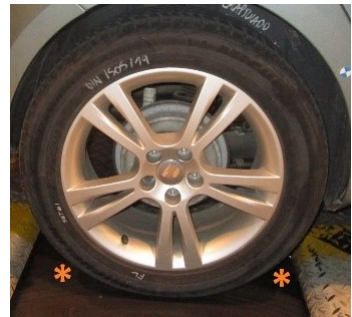

$4 \mathrm{kHz}$

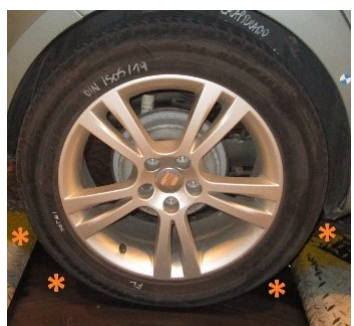

$4 \mathrm{kHz}$

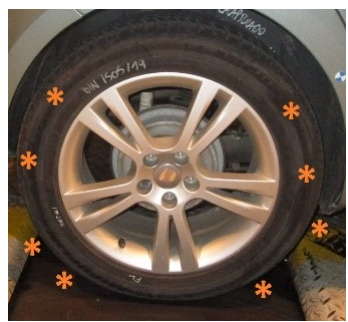

$4 \mathrm{kHz}$

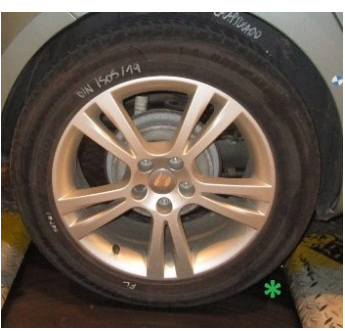

Frequency-averaged

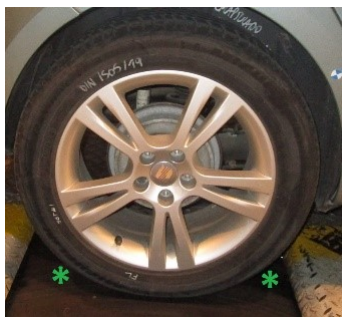

Frequency-averaged

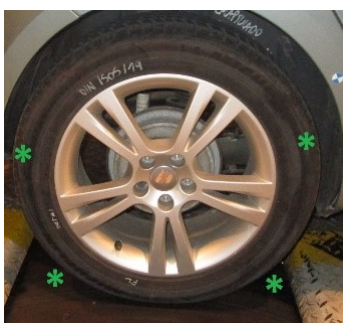

Frequency-averaged

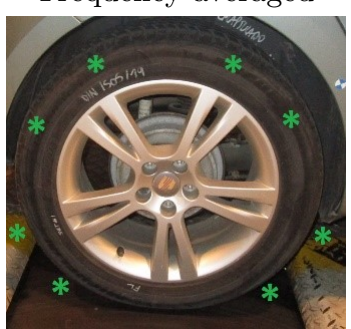

Frequency-averaged

Figure 8: Equivalent source positions using the frequency dependent $\ell_{1}$ norm regularisation at $300 \mathrm{~Hz}, 1 \mathrm{kHz}$ and $4 \mathrm{kHz}$ respectively (orange stars) and the frequency averaged $\ell_{1}$ norm regularisation (green stars) for (a) 1 , (b) 2 , (c) 4 and (d) 8 equivalent sources 
As expected, it is shown that the $\ell_{1}$ norm regularisation at each frequency delivers frequency dependent source distributions. For a small number of sources, the source positions are generally concentrated towards the leading and trailing edge of the tyre, where the tyre noise phenomena are known to be dominant, while using a higher number of sources spreads their distribution along the tyre circumference. It is also seen that the distance between the frequency dependent source positions decreases with increasing frequency. This is expected to render a well-conditioned inverse within the inverse problem, since it has been suggested that the distance between two equivalent sources $d$ should not be larger than half the wavelength of the maximum frequency $\lambda_{\max }\left(d \leq \lambda_{\max } / 2\right)$ [18]. The source positions chosen using the frequency-averaged $\ell_{1}$ norm regularisation, on the other hand, are expected to prioritise the reconstruction at frequency lines where the response is the highest, since the cost function to be minimised is the sum of the least-squares residuals at the various frequencies. The agreement between the fixed positions and the corresponding frequency dependent ones at $300 \mathrm{~Hz}$ and at $1 \mathrm{kHz}$ and the disagreement at $4 \mathrm{kHz}$ indicates that the source strengths at $300 \mathrm{~Hz}$ and $1000 \mathrm{~Hz}$ are 'predominant' and take over those at $4 \mathrm{kHz}$ when the frequency-averaged regularisation is used. In Fig. 9, the condition number of $\mathbf{G}_{\text {near }}^{\prime}$ for 4 and 8 equivalent sources is given over the frequency range of interest using the distributions suggested by the two $\ell_{1}$ norm regularisation techniques.

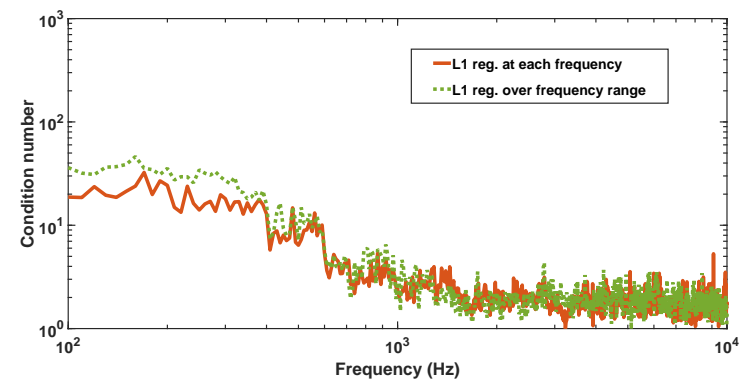

(a)

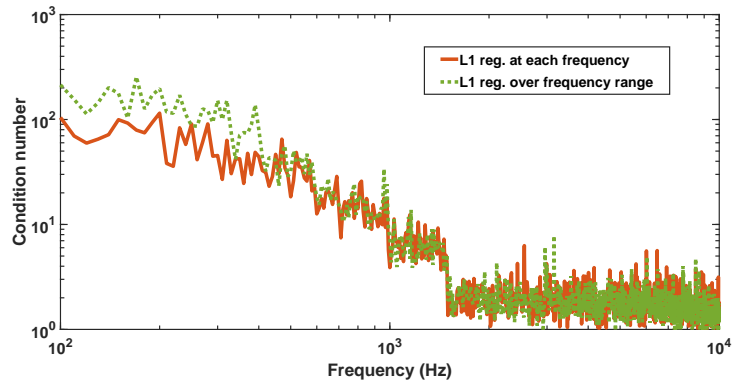

(b)

Figure 9: Condition number of $\mathbf{G}_{\text {near }}^{\prime}$ as a function of frequency for (a) 4 and (b) 8 equivalent sources.

The decrease of the distance between the equivalent sources with increasing frequency, using the $\ell_{1}$ norm source elements are small compared to a wavelength and the condition number normally rises. However, both techniques deliver source geometries with acceptable conditioning. In Fig. 10, the computed SPL in $1 / 3$ octave bands at far field microphone No. 5 is given over the frequency range of interest for the various numbers of equivalent sources chosen with the two methods and compared to the SPL measured directly.

The frequency - averaged formulation for the equivalent source positions in Eq. (4) was used even for the $1 / 3$ octave results, but in this case with the averaging only over the frequency bins in each $1 / 3$ octave. The results for microphone No. 5 in Fig. 3(a) is chosen as it is close to the middle of the far field array and subject to the highest acoustic response, while the SPL is given in $1 / 3$ octave bands in order to avoid the fluctuations of the broadband tyre noise, especially at higher frequencies, to thus better visualise the and approximately $2.5 \mathrm{kHz}$ above which the SPL decreases, while the most dominant region is between 450 $\mathrm{Hz}$ and $1 \mathrm{kHz}$. For a small number of sources, the deviations between the directly measured spectra and the synthesised responses at high frequencies are due to insufficient sources to synthesise the sound field. Using the inverse method with a single source element, as in Fig. 10(a), is equivalent to focusing a beamformer norm regularisation techniques give good estimates of the radiated pressure up to $750 \mathrm{~Hz}$, above which the pressure is underestimated. The use of 2 sources extends the accuracy of the synthesis up to approximately $1.5 \mathrm{kHz}$ above which the number of sources is not sufficient for an accurate reconstruction. By using 4 and 8 equivalent sources, this underestimation at higher frequencies is largely taken care of. However, an overestimation of the response at low frequencies then becomes apparent. The condition numbers in Fig. 9 suggest that the errors in the radiated pressure spectra become significant when the condition number of 


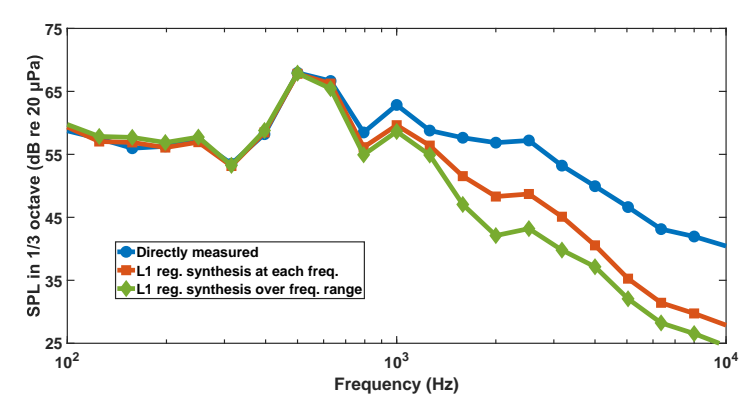

(a)

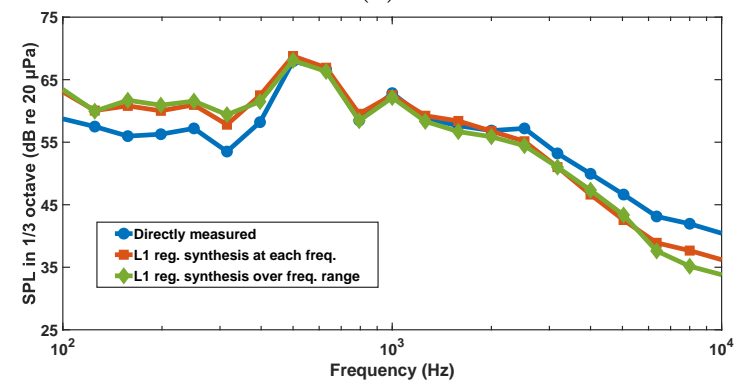

(c)

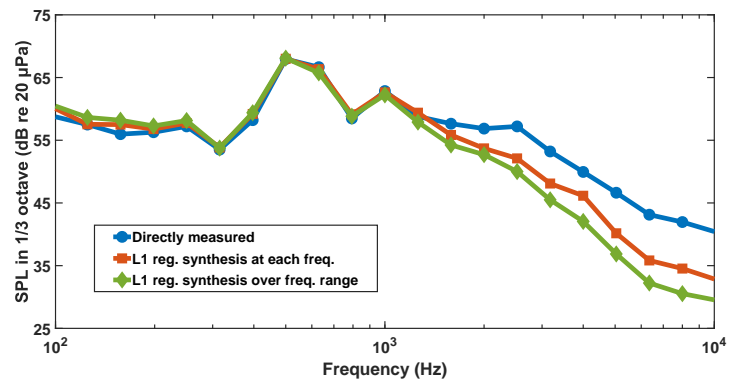

(b)

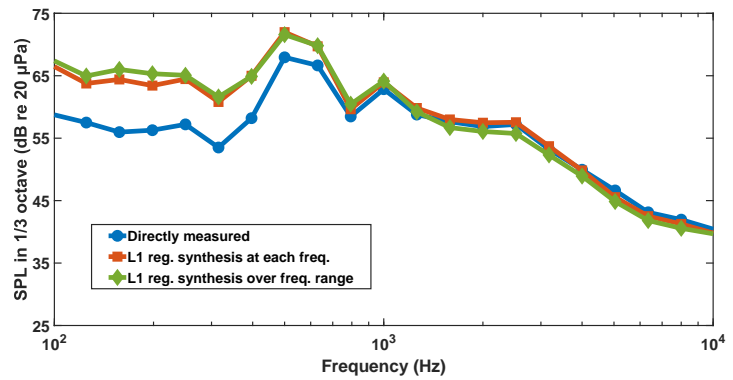

(d)

Figure 10: SPL in 1/3 octave bands at far field microphone 5 over the frequency range for (a) 1 equivalent source, (b) 2 equivalent sources, (c) 4 equivalent sources and (d) 8 equivalent sources

$\mathbf{G}_{\text {near }}^{\prime}$ exceeds about 10 to 15, which implies a relatively high sensitivity of the result to the conditioning. In Fig. 11, the A-weighted SPL at each microphone in the far field microphone array is shown, for the various number of equivalent sources selected using the two $\ell_{1}$ norm regularisation strategies.

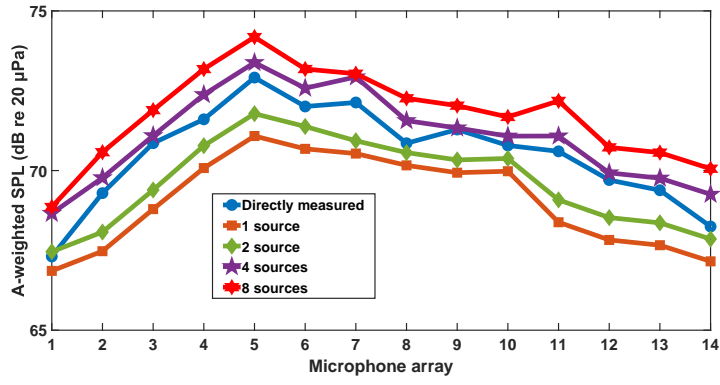

(a)

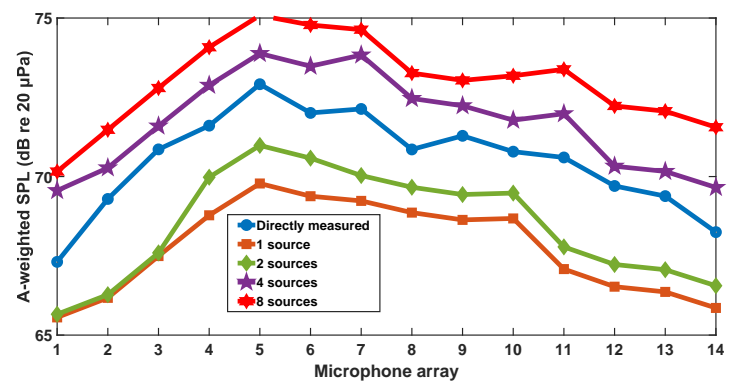

(b)

Figure 11: A- weighted SPL across the far field array for various number of sources using (a) $\ell_{1}$ norm regularisation at each frequency and (b) $\ell_{1}$ norm regularisation over the whole frequency range

The use of $\ell_{1}$ norm regularisation at each frequency (Fig. 11(a)) shows that acceptable representations of the directly measured spectra can be synthesised, especially when using 4 sources with $0.5-1 \mathrm{~dB}$ deviations. This accuracy is partly achieved due to the application of the A-weighting in the overall spectra which discriminates against the low frequency components where the errors are more important for the 4-element case. In Fig. 11(b), however, the demand for fixed source positions increases the deviation using 1,2 or 8 sources to an average of 3-5 dB, but it is shown that in the 4 - element case, a synthesised curve with an average deviation of $1.5-2 \mathrm{~dB}$ is achieved.

This deviation can be decreased by using a different number of sources within different frequency ranges. For example, the synthesised spectra shown in Fig. 10 suggest that, for the fixed source position synthesis, 
using a combination of 2 sources up to $1 \mathrm{kHz}$ and 8 sources from $1 \mathrm{kHz}$ upwards should yield best results over the frequency range of interest. However, improved results can be achieved using the same number of fixed sources over the frequency range by applying a conventional $\ell_{2}$ norm regularisation at $\mathbf{G}_{\text {near }}^{\prime}$, as formulated in Eq. (6) to alleviate the effect of the bad conditioning at low frequencies.

\subsection{Effect of additional $\ell_{2}$ norm regularisation}

As stated in Section 2.3, the $\ell_{2}$ norm regularisation discriminates against the smallest singular values of $\mathbf{G}_{\text {near }}^{\prime}$ by using a parameter $\beta$. The degree to which this regularisation affects the singular values depends upon their magnitudes and, in particular, the number of singular values with magnitudes below the threshold determined by $\beta$. Thus, with a carefully chosen parameter only the singular values at frequencies at which the condition number is high are affected [18]. A number of techniques have been documented for selecting the optimum value for the regularisation parameter $\beta$ [17]. Herein, a trial-and-error strategy is first followed and the selected parameter is the one which gives the minimum least squares error over the frequency range of interest, as defined in Eq. (6). The L-curve and the generalised cross-validation methods are then used to verify the choice of the regularisation parameter. The techniques are used only in the 4 and 8 -source case, where errors occur due to the poor low frequency conditioning. Using the regularisation for a smaller number of sources has no effect on the result, as the errors in those cases are due to the underestimation of the tyre sound field at high frequencies which can only be solved by increasing the number of sources. In Fig. 12, the sum of the least squares errors over the frequency range is given using different regularisation parameters $\beta$.

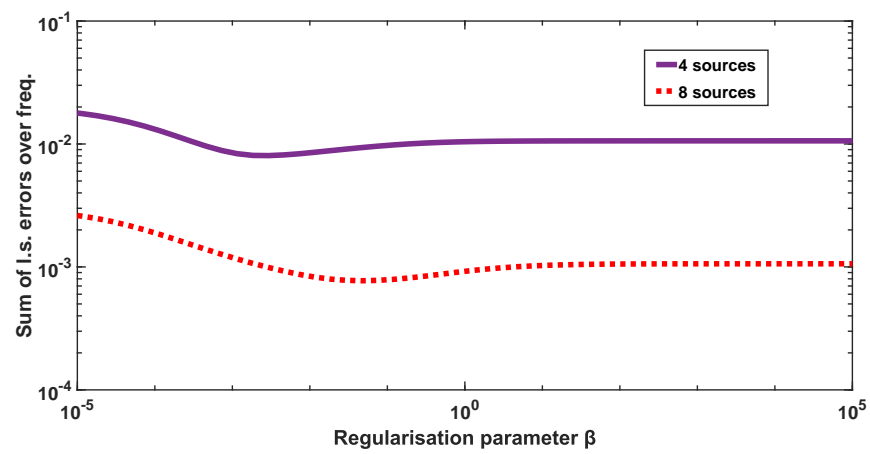

Figure 12: Sum of the least squares error over the whole frequency range for different regularisation parameters $\beta$ using 4 and 8 fixed sources

For very small values of $\beta$, the effect of the regularisation is not adequate and does not manage to alleviate the effect of the bad conditioning. For higher values, the low frequency conditioning is improved at the expense of a slight decrease at high frequency spatial resolution which incurs errors in the synthesised spectra. For the 4 -source case the optimum $\beta$ is seen to be close to 0.002 while for the 8-source case close to 0.03. The same trends are seen when using the L-curve and generalised cross-validation with the optimum values found to be almost identical to the ones retrieved by trial-and-error.

The coherence between the equivalent source strengths can be calculated from the cross-spectral density and power spectral density terms that form the elements of Eq. 9. For the experiments reported here, the coherence between the equivalent source strengths varied from 0 to about 0.8 , depending on frequency, with the coherence generally being higher at lower frequencies. The non-zero coherence indicates that the equivalent source strengths are not entirely uncorrelated, particularly at low frequencies.

Fig. 13 shows the $\ell_{1}$ norm regularised synthesised spectra over frequency for 4 and 8 sources before and after the use of the $\ell_{2}$ norm regularisation. It is found that for both cases the low and mid frequency errors due to the bad conditioning are lessened without significant decrease of spatial resolution at high frequencies. Correspondingly, in Fig. 14, the updated A-weighted SPL across the entire microphone array is given, using 4 and 8 fixed equivalent sources after the $\ell_{2}$ norm regularisation, while the A-weighted SPL curves using 1 


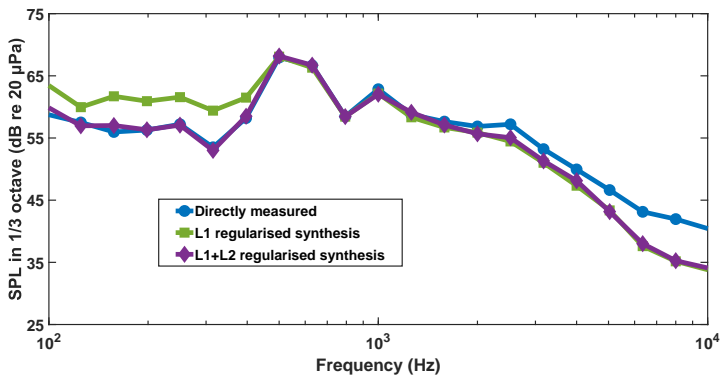

(a)

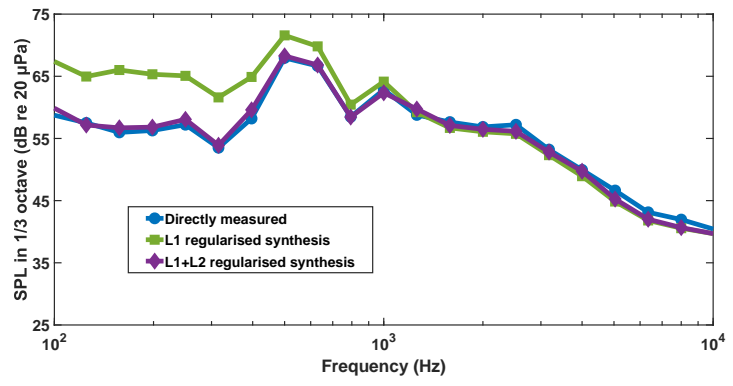

(b)

Figure 13: SPL in 1/3 octave bands at far field microphone 5 over the frequency range before and after the use of $\ell_{2}$ norm regularisation compared to the directly measured spectra for (a) 4 and (b) 8 equivalent sources

and 2 sources are repeated to summarise the final results of the proposed methods. The application of the $\ell_{2}$ norm regularisation is not shown in Fig. 14 as it does not influence the synthesis results.

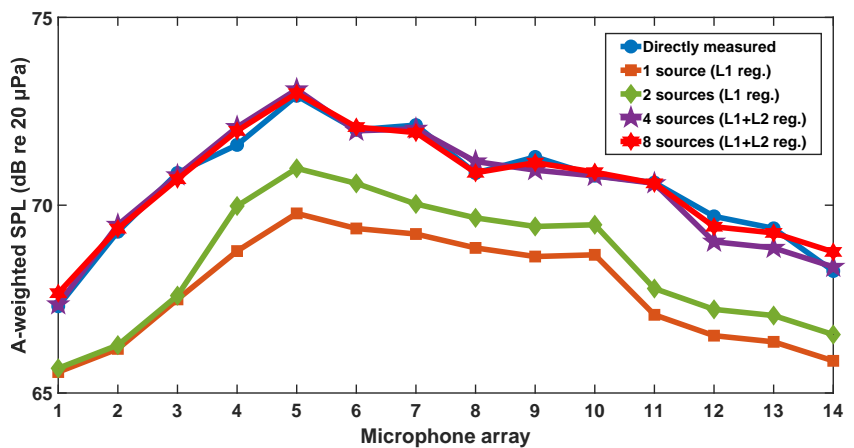

Figure 14: A-weighted SPL across the far field array using 1, 2, 4 and 8 fixed sources over frequency compared to the directly measured spectra

The synthesised responses are compared to the directly measured SPL. It can be seen that using 4 or 8 sources gives a good representation of the directly measured SPL. The response acquired by using both $\ell_{1}$ norm and $\ell_{2}$ norm regularisation for 4 fixed sources is slightly underestimated towards the right end of the array, while the synthesised response using 8 fixed sources after $\ell_{1}$ norm and $\ell_{2}$ norm regularisation is the most accurate, providing an average deviation of $0.3 \mathrm{~dB}$. It is thus seen that the far field spectra can be accurately synthesised using fixed source positions over the frequency range of interest by applying an additional $\ell_{2}$ norm regularisation at the 4 and 8 -element case to tackle the bad low frequency conditioning. Therefore, using 4 fixed sources over the frequency range of interest appears to be the optimum strategy in terms of both minimising the measurement complexity and delivering accurate representations of the directly measured spectra.

In other experiments, at higher road speeds and with the engine idling, the 4 fixed source strategy has also been found to give good representations of the far field pressure with similar source positions. This indicates that this result is reasonably robust, although further work is required to expore this in more detail.

\section{Conclusions}

In this paper, the application of a number of regularisation techniques associated with the use of the inverse method to the indoor tyre pass-by noise synthesis is evaluated experimentally. Pressure spectra measured very close to a car tyre rolling at $50 \mathrm{~km} / \mathrm{h}$ with the engine off are used, together with measured 
transfer responses between the near field microphone array and a number of candidate equivalent source positions to obtain an equivalent source distribution. Two different strategies are followed to select a limited number of these equivalent source positions. The first one utilises an $\ell_{1}$ norm regularisation technique to optimise the positioning of the equivalent sources at each frequency, which results in frequency dependent source positions. The second utilises a generalisation of this $\ell_{1}$ norm regularisation technique over a range of frequencies to obtain fixed equivalent source positions that are frequency independent. The source distributions selected by the two methods are used, together with measured transfer responses between a far field microphone array and the source positions, to synthesise the response at the far field and this is then compared with the directly measured pressures.

Using the $\ell_{1}$ norm regularisation at each frequency gives a fairly accurate A-weighted SPL estimate across the microphone array, with an average deviation of $1 \mathrm{~dB}$, when 4 equivalent sources with frequency dependent source positions are assumed. However, considering the need for measurements at the various positions selected at each frequency bin, the measurement complexity is significantly increased.

On the other hand, the $\ell_{1}$ norm regularisation over the frequency range of interest produces fixed source positions, at the expense of the synthesis accuracy. Using 1 and 2 equivalent sources results in an underestimation of the field at high frequencies which can only be tackled by increasing the number of sources. Using 4 or 8 sources improves the high frequency synthesis, but at the expense of an overestimation of the field at low frequencies, which is due to the effect of the poor low frequency conditioning. This is taken care of by using an additional $\ell_{2}$ norm regularisation technique which alleviates the effect of the bad low frequency conditioning and improves the synthesised responses at low frequencies when 4 and 8 sources are used.

The additional use of the $\ell_{2}$ norm regularisation for the 4 and 8 - fixed source case delivers A-weighted SPL estimates of the pressure of the far field microphones with average deviations of 0.5 and 0.3 respectively. Considering the need to minimise the measurement instrumentation and complexity, the 4 fixed source strategy is thus identified as the best engineering trade-off between complexity and accuracy. It is noted that these results are valid for $50 \mathrm{~km} / \mathrm{h}$ rolling speed at which the pressure data and transfer responses were measured and further work is needed to assess their validity in different operational conditions.

\section{Acknowledgements}

The authors gratefully acknowledge the European Commission for its support of the Marie Sklodowska Curie program through the ETN PBNv2 project (GA 721615). The authors would like to thank ISVR Consulting for the loan of the volume velocity source and both Juan J. Garcia and Xavier M. Clos for their valuable help in the measurements at IDIADA.

\section{References}

[1] International Organization for Standardization, ISO 362-3:2016: Measurement of noise emitted by accelerating road vehicles-Engineering method-Part 1: $\mathrm{M}$ and $\mathrm{N}$ categories.

[2] K. Janssens, P. Aarnoutse, P. Gajdatsy, L. Britte, F. Deblauwe, H. Van der Auwerauer, Time-domain source contribution analysis method for in-room pass-by noise, SAE Tech. Pap. 2011-01-1609, 2011.

[3] J. W. Verheij, Inverse and reciprocity methods for machinery noise source characterization and sound path quantification. Part 1: Sources, Int. J. Acoust. Vib. 2 (1) 11-20, 1997.

[4] J. W. Verheij, Inverse and reciprocity methods for machinery noise source characterization and sound path quantification. Part 2: Transmission Paths, Int. J. Acoust. Vib. 2 (3) 103-112, 1997.

[5] Fleszar, A. R., van der Linden, P. J. G., Johnson, J. R., Grimmer, M. J., Combining vehicle and test-bed diagnosis information to guide vehicle development for pass-by noise, SAE Tech. Pap. 2001-01-1565; 2001.

[6] Genuit K, Guidati S, Sottek R., Progresses in pass-by simulation techniques, SAE Tech. Pap. 2005-01-2262; 2005.

[7] Kim, B. K., Yoo, S. W., Kim, H. J., Zwanzig, K., Prediction of vehicle pass-by noise using indoor measurement, SAE Tech. Pap. 2001-01-1563; 2001.

[8] Ryu, Y., Schumacher, A., Hirayama, M., Shirahashi, Y., Contribution analysis of exterior noise with indoor pass-by measurement, SAE Tech. Pap. 2011-26-0062; 2011.

[9] J. Putner, M. Lohrmann, H. Fastl, Contribution analysis of vehicle exterior noise with operational transfer path analysis', Proc. ICA 2013, Montreal, Canada, 2013.

[10] Z. Chu, H. Wang, C. Chen, H. Yan, R. Kang, Source Path Contribution Analysis for Vehicle Indoor Pass-By Noise, SAE Tech. Pap. 2017-01-2247, 2017. 
[11] Goto, K., Kondo, T., Takahira, M., Umemura, E., Komada, M., Nishimura, Y., Indoor Pass-By Noise Evaluation System Capable of Reproducing ISO Actual Road Surface Tire Noise, SAE Tech. Pap. 2016-01-0479; 2016.

[12] W. Kropp, K. Larsson, F. Wullens, P. Homstad, F.X. Becot, The generation of the tyre/road noise', Proc. ICSV 2003, Stockholm, Sweden, 2003.

[13] W. Hendricx, F. Mancosu, Experimental determination of the noise emitting parts of a rotating tire in the European research project TINO, J. Passeng. Cars 108 (6) 2717-2721, 1999.

[14] P.A. Morgan, S.M. Phillips, G.R. Watts, The localisation, quantification and propagation of noise from a rolling tyre, TRL Limited, Published Project Report PPR 138, 2006.

[15] D. Berckmans, P. Kindt, P. Sas, W. Desmet, Evaluation of substitution monopole models for tire noise sound synthesis, Mech. Syst. Signal Process. 24 (2010) 240-255.

440 [16] P.A. Nelson, S.H. Yoon, Estimation of acoustic source strength by inverse methods: Part I: Conditioning of the inverse problem, J. Sound Vib. 233 (4) 643-668, 2000.

[17] S.H. Yoon, P.A. Nelson, Estimation of acoustic source strength by inverse methods: Part II: Investigation of methods for choosing regularisation parameters, J. Sound Vib. 233 (4) 669-705, 2000.

[18] K.R. Holland, P.A. Nelson, The application of inverse methods to spatially-distributed acoustic sources, J. Sound Vib. 332 5727-5747, 2013

[19] Y. Kim, P.A. Nelson, Spatial resolution limits for the reconstruction of acoustic source strength by inverse methods, J. Sound Vib. 265 583-608, 2003.

[20] M. Elad, Sparse and redundant representations: From theory to applications in signal and image processing, Springer, New York, 2010.

21] S. Foucart, H. Rauhut, A mathematical introduction to compressive sensing, Springer, New York, 2013.

[22] G. Chardon, L. Daudet, A. Peillot, F. Ollivier, N. Bertin, R. Gribonval, Near-field acoustic holography using sparse regularisation and compressive sampling principles, J. Acoust. Soc. Am. 132 (3) 1521-1534, 2012.

[23] J. Hald, A comparison of iterative sparse equivalent source methods for near-field acoustic holography, J. Acoust. Soc. Am. 143 (6) 3758-3769, 2018.

[24] E. Fernandez-Grande, A. Xenaki, P. Gerstoft, A sparse equivalent source method for near-field acoustic holography, J. Acoust. Soc. Am. 141 (1) 532-542, 2017.

[25] T. Suzuki, L1 generalised inverse beamforming algorithm resolving coherent/incoherent, distributed and multipole sources, J. Sound Vib. 330 5835-5851, 2011.

[26] D.M. Malioutov, M. Cetin, A.S. Willsky, A sparse single reconstruction perspective for source localisation with sensor arrays, IEEE Trans. Signal Process. 53 (8) 2477-2488, 2005.

[27] S. Cotter, B. Rao, K. Engan, K. Kreutz-Delgado, Sparse solutions to linear inverse problems with multiple measurement vectors, IEEE Trans. Signal Process. 53 (7) 2477-2488, 2005.

[28] E. van den Berg, M.P. Friedlander, Theoretical and empirical results for recovery from multiple measurements, SIAM J. Sci. Comput. 21 (4) 1201-1229, 2011.

465 [29] J. Tropp, Algorithms for simultaneous sparse approximation. Part II: Convex relaxation, Signal Process. 86 589-602, 2006.

[30] J. Chen, X. Huo, Theoretical results on sparse representations of multiple measurement vectors, IEEE Trans. Signal Process. 54 (12) 4634-4643, 2006.

[31] Shin K. and Hammond J., Fundamentals of Signal Processing for Sound and Vibration Engineers, Wiley Press, Chichester, UK, 2008.

470 [32] M. Grant, S. Boyd, CVX: MATLAB software for disciplined convex programming (version 2.1), http://cvxr.com/cvx.

[33] E. van den Berg, M.P. Friedlander, http://www.cs.ubc.ca/labs/scl/spgl1, SPGL1: A solver for large-scale sparse reconstruction.

[34] E. van den Berg, M.P. Friedlander, Sparse optimisation with least-squares constraints, SIAM J. Sci. Comput. 21 (4), 2011 1201-1229.

5] E. van den Berg, M.P. Friedlander, Theoretical and empirical results for recovery from multiple measurements, IEEE Trans. Inf. Theory 56 (5) 2516-2527, 2010.

[36] https://www.applusidiada.com/en/.

[37] https://www.isvr.co.uk/automotive/sound-sources.htm.

[38] K.R. Holland, P.A. Nelson, An experimental comparison of the focused beamformer and the inverse method for the characterisation of acoustic sources in ideal and non-ideal acoustic environments, J. Sound Vib. 331 4425-4437, 2012. 